\title{
Numeral legibility and visual complexity
}

\author{
BEIER Sofie ${ }^{a^{*}} ;$ BERNARD Jean-Baptiste $^{\mathrm{b}}$ and CASTET Eric ${ }^{\mathrm{c}}$ \\ a The Royal Danish Academy of Fine Arts, Denmark \\ ${ }^{\mathrm{b}}$ Aix-Marseille Université \& Laboratoire de Psychologie Cognitive \& Fondation de l'Avenir - Visaudio \\ c Aix-Marseille Université \& Laboratoire de Psychologie Cognitive \\ * Corresponding author e-mail: sbe@kadk.dk \\ doi: $10.21606 / \mathrm{dma} .2017 .246$
}

\begin{abstract}
To enhance the peripheral legibility of numerals we designed three versions of the digits from 1 through 9 by modifying the complexity of each numeral (equivalent to their digit skeleton) while controlling for variations in other physical parameters. Observers identified the different versions of the digits in random three-digit strings, presented within their peripheral visual field. Our results showed that the digit ' 1 ' should have a narrow design without a crossbar at the bottom, the digits ' 3 ' and ' 9 ' should benefit from open apertures, and the digit ' 7 ' should have a straight leg and no serif at the horizontal bar. The data further demonstrated that crowded digits presented in the periphery of the visual field generally profit from a short morphological skeleton. The findings can improve the identifiability of numbers for readers with normal visions as well as for readers with central visual field loss.
\end{abstract}

Typefaces, numerals, legibility, inclusive design

\section{Introduction}

If a reader misreads a number on a road sign, a medicine information leaflet, or an aircraft display, the potentially flawed action which follows can have severe consequences. With this in mind, it is important to realise that few studies in the research literature concern numeral legibility. By identifying visual factors influencing numeral legibility, we seek to add new knowledge that could benefit both visually impaired readers and readers with normal vision. The findings could help type designers create legible digits, and could also help graphic designers determine which typeface to choose when maximum legibility is a priority. Among possible limiting visual factors, we were interested in studying the effect of the length of the numeral skeleton on numeral legibility. This is based on previous studies showing the effect of letter skeleton length (potentially measuring letter complexity) on peripheral letter legibility (Bernard \& Chung, 2011; Wang et al., 2014). 


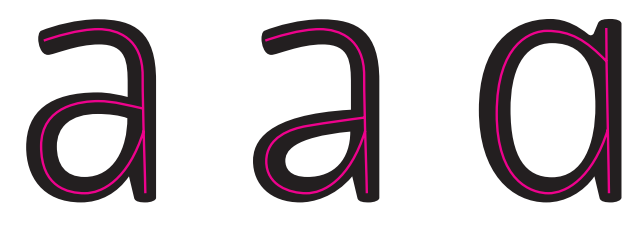

Figure 1: The skeleton of a letter or digit is the basic structure of the character. In this illustration, the letter skeleton varies while other parameters, such as stroke weight and width, are identical among the tree letters.

Whilst identifying a letter within a word, the reader will draw on a mental library of all the words he or she has been exposed to before (Legge, Klitz, \& Tjan, 1997). This means that when a reader encounters an illegible letter, he/she can draw on information from adjacent letters and from the sentence structure, and thus make an educated guess of what the letter might be (Pelli \& Tillman, 2007). This is rarely possible when the target is a digit. In such situations, there will be little or no additional help from the surrounding digits or the structure of the text. It is therefore essential to prevent one digit being mistaken for another (Figure 2). This pertains especially to specific visual conditions that make numerals difficult to identify. For instance, letter/numeral recognition is harder for small print sizes near the acuity limit because of human optical and neural limitations. When readers cannot use their central vision (such as patients with age-related macular degeneration (ARMD)), symbol recognition can be difficult, even for large print sizes. This is due to visual crowding (Pelli et al., 2004), a phenomenon which impairs symbol recognition when a symbol is surrounded by other symbols in the peripheral visual field. As previously explained, patients with ARMD, unfortunately, cannot rely on the general context to improve their limited numeral recognition performance.
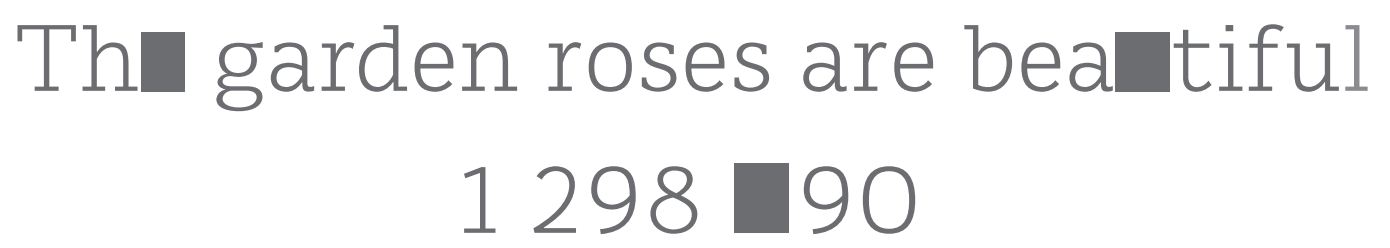

Figure 2: Based on word and sentence structure, it is possible to guess the missing letters in the top row. However, there is no way to guess the missing number in the bottom row.

The so-called alphanumeric category effect (Hamilton, Mirkin, \& Polk, 2006; Jonides \& Gleitman, 1972; Polk \& Farah, 1998) describes the fact that in a different-category target search, subjects tend to have a longer reaction time when detecting a letter among letters than when detecting a letter among digits, and vice versa. This suggests that digits and letters are, to some degree, independently processed. Yet, there are indications that this difference is related to habit. As readers often perceive letters and digits under separate circumstances, it might be more difficult to process them when they are presented collectively. This idea is demonstrated by Polk and Farah (1998), who found that the alphanumeric category effect is less evident among Canadian postal workers, who have a daily routine of sorting postal codes of mixed letters and digits, and by Jonides and Gleitman (1972), who found that results were affected by whether observers perceived 0 as a digit (zero) or as a letter.

If the phenomenon is due to habit alone, the identification of letters and digits should be equally difficult. That is, however, not the case. There is substantial evidence suggesting a numeral identification advantage, with studies demonstrating that it is easier to identify digits than letters (Schubert, 2016). Further, the vast amount of literature on pure alexia showed that digit naming can be less impaired than letter naming in certain patients (Starrfelt \& Behrmann, 2011). In fact, cases of digit naming impairment with intact letter naming impairment have yet to be reported (Rath et al., 2015).

One reason for this could be related to the difference in the visual properties of letters and digits. To investigate this hypothesis, Starrfelt and Behrmann (2011) visually overlapped lowercase letters and 
digits in the typefaces Times and Arial. They suggested that as there are more letters in the alphabet than digits, letters have a larger number of possible competitors, and hence, single symbol identification should be more difficult for letters than for digits. Schubert (2016) focused on scenarios where letters and digits are mixed. She used uppercase letters and digits of four different typefaces, separating the character features into different units such as 'slant', 'curve' and 'orthogonal'. While overlapping two characters, she considered position and relative size and found no indications that digits have more distinctive forms than letters. However, a curve within a typeface can vary highly between characters (Figure 3).

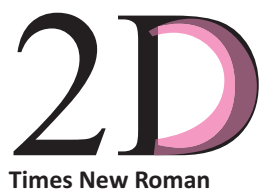

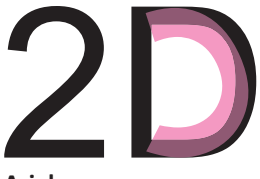

Arial

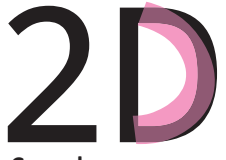

Consolas

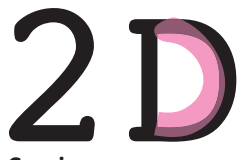

Courier

Figure 3: The four typefaces applied in the study by Schubert (2016). To demonstrate that curves within a typeface can vary significantly between characters, the curve of the ' 2 ' has been rotated and scaled to fit the curve of the ' $D$ '.

It is also possible that the numeral identification advantage is related to a difference in letter and digit structure that cannot be detected by measuring the physical overlap of shapes. While uppercase and lowercase letters originate in the Roman capitals and the Carolingian minuscule, numerals are HinduArabic. This difference in origin has left a mark on the basic structure of letters and digits. Roman capital letters were originally cut in stone, and the letter shapes are therefore dominated by straight horizontal, vertical, and diagonal strokes mixed with clear circular strokes. The vertical stroke survived in lowercase letters, through the cursive tradition of connecting the downstroke with the upstroke of the following letter. About $62 \%$ of lowercase letters and about $65 \%$ of uppercase letters have a vertical stroke. Compared to this, only $20 \%$ of digits have a vertical stroke (Figure 4 ). It appears that the downward-upward stroke in lowercase letters contribute to a steady rhythm when the letters are put into words and sentences (Johnston, 1913).

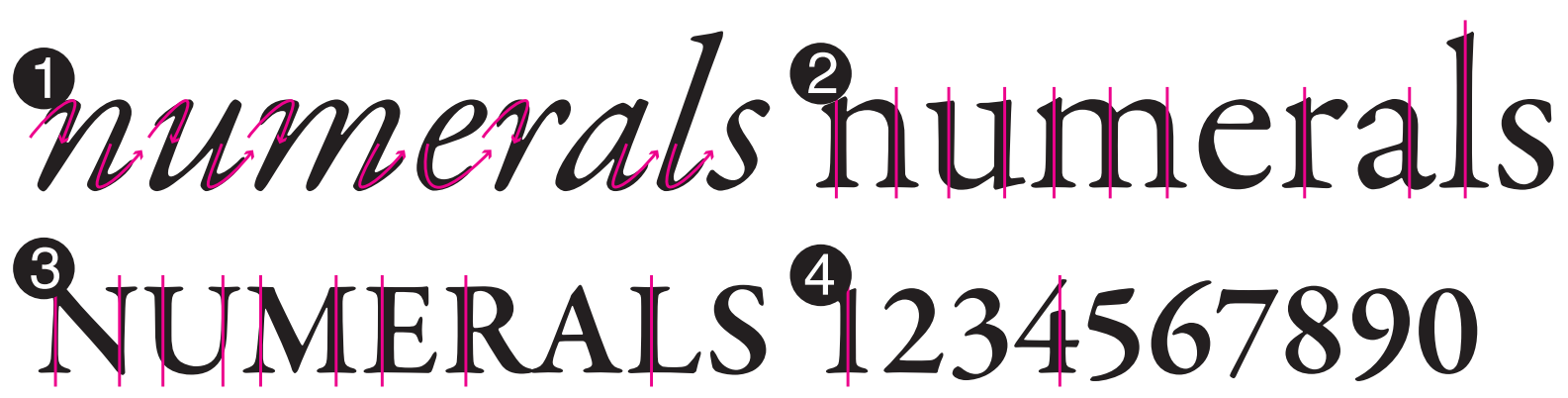

Figure 4: 1) The cursive writing hand that connects downward and upwards strokes. 2) The vertical strokes of lowercase letters. 3) The vertical strokes of uppercase letters. 4) The vertical strokes of numerals. Demonstrated in the typeface Garamond Premier Pro.

The oft-repeated saying that 'type is a beautiful group of letters, not a group of beautiful letters' (Carter, 2004), suggests that letters should be designed to be parts of words, not individual units. That is the essential difference between letters and digits. Since each digit represents a number, their functions are independent of other symbols. That is not the case for letters. Except for rare exceptions (for instance, the ' $a$ ' and ' $i$ ' in the English language), single letters are only abstract symbols with no numerical value or semantic meaning. It is when letters are flanked by other letters that they fulfil their purpose by forming words. Following this, matters related to word readability and the flow of reading are less relevant in the study of numerals. Research into letter legibility can, however, also provide useful information for optimising the legibility of numerals. 


\section{Experiment: the skeleton structure of the digits}

Previous research into the foveal and peripheral legibility of numerals have aimed at reinventing the shapes (Lansdell, 1954), improving the shapes of seven-segment numerals (Van Nes \& Bouma, 1980), or at comparing the digits of different typefaces (Berger, 1944; Fox, Chaparro, \& Merkle, 2008; Hind, Tritt, \& Hoffmann, 1976; Smuc, Windhager, Siebenhandl, \& Egger, 2007).

Within typeface legibility research there is a tendency to seek answers by comparing different typefaces in psychophysical experiments. The problem with such an approach is that it is difficult to isolate one visual feature from another, as different typefaces have different proportions, weights, contrasts, and styles (Beier, 2016). That makes it difficult to interpret the findings of such studies, as there are too many typographical variables at play at once. Here, we decided to focus on visual complexity, a factor that has been shown to influence letter legibility. As several studies have suggested a link between the visual complexity of symbols and their skeleton length, we chose to investigate the effect of the skeleton length of a numeral on its legibility. We measured peripheral legibility, a way to investigate directly how we could improve numeral recognition performance in patients with central field loss.

\subsection{Subjects}

Five subjects (two females and three males) with normal or corrected-to-normal vision aged from 21 to 38 years participated in this study. The subjects were students and post-docs from the Aix-Marseille Université. They were paid 10 euros each for their participation in the experiment. The research followed the tenets of the Declaration of Helsinki and was approved by the Ethical Committee for Protection of Human Subjects at the Aix-Marseille Université. Written informed consent was obtained from each subject after the nature and purpose of the experiment had been explained.

\subsection{Apparatus}

Stimuli were displayed on a 21-inch CRT color monitor (ViewSonic P227f, refresh rate $=120 \mathrm{~Hz}$, resolution $=1152 \times 854$ pixels) driven by a Windows computer running custom software developed in Python with the Psychopy library. The subjects sat in a comfortable chair with their eyes at a distance of $40 \mathrm{~cm}$ from the monitor in a dimly lit room (screen visual angle: $50.8^{\circ} \times 37.7^{\circ}$ ). An eye tracker (Eyelink 1000 Tower Mount distributed by SR Research Ltd., Mississauga, Ont., Canada) was connected to our system to control the gaze fixation of the subjects. Numerals were displayed in black (luminance: $0.3 \mathrm{~cd} / \mathrm{m} 2$ ) on a light grey background (luminance: $60 \mathrm{~cd} / \mathrm{m} 2$ ).

\subsection{Design of the numerals}

For this experiment, we isolated the variables under investigation by altering one visual feature at a time. By keeping the test material within one typeface, we can ensure that the findings are related solely to the matter under investigation. For this purpose, we extended the typeface DejaVu Sans to contain three variations of each of the numerals from 1-9.

Figure 5 shows the different versions of each numeral. For the numbers 1 and 8 , one aspect of interest was the effect of character width; Fox et al. (2008) found an advantage of a wider ' 1 ', and Berger (1944) and Smuc et al. (2007) both recommended narrow versions of ' 8 '. To control the variables, the only difference between $1 a$ and $1 b$ and between $8 v$ and $8 x$ is the width. We were further interested in the effect of a cross bar on the numbers ' 1 ' and ' 4 '; the open and close counter of the numbers ' 2 ', ' 3 ', ' 5 ' and ' 9 '; the $x$-height of the number ' 6 '; and the cross sections of the numbers ' 2 ' and ' 7 '. 


\begin{tabular}{|c|c|c|c|}
\hline ID & a & b & $c$ \\
\hline digit & $\mathbf{1}$ & $\mathbf{1}$ & $\mathbf{1}$ \\
\hline $\begin{array}{c}\text { varia } \\
\text { ble }\end{array}$ & narrow & wide & $\begin{array}{c}\text { cross } \\
\text { bar }\end{array}$ \\
\hline
\end{tabular}
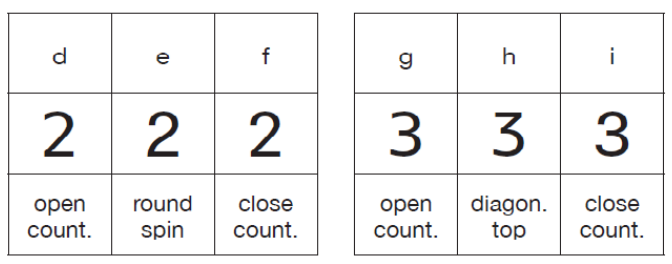

\begin{tabular}{|c|c|c|c|}
\hline ID & j & k & l \\
\hline digit & $\mathbf{4}$ & $\mathbf{4}$ & $\mathbf{4}$ \\
\hline $\begin{array}{c}\text { varia } \\
\text { ble }\end{array}$ & $\begin{array}{c}\text { close } \\
\text { top }\end{array}$ & $\begin{array}{c}\text { open } \\
\text { top }\end{array}$ & $\begin{array}{c}\text { cross } \\
\text { bar }\end{array}$ \\
\hline
\end{tabular}

\begin{tabular}{|c|c|c|}
\hline$m$ & $n$ & $\circ$ \\
\hline 5 & 5 & 5 \\
\hline $\begin{array}{c}\text { open } \\
\text { count. }\end{array}$ & $\begin{array}{c}\text { close } \\
\text { count. }\end{array}$ & $\begin{array}{c}\text { square } \\
\text { loop }\end{array}$ \\
\hline
\end{tabular}

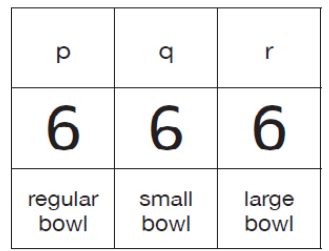

\begin{tabular}{|c|c|c|c|}
\hline ID & s & t & u \\
\hline digit & 7 & 7 & 7 \\
\hline $\begin{array}{c}\text { varia } \\
\text { ble }\end{array}$ & $\begin{array}{c}\text { straight } \\
\text { leg }\end{array}$ & $\begin{array}{c}\text { bended } \\
\text { leg }\end{array}$ & $\begin{array}{c}\text { serif } \\
\text { at top }\end{array}$ \\
\hline
\end{tabular}

\begin{tabular}{|c|c|c|}
\hline$v$ & $w$ & $x$ \\
\hline $\mathbf{8}$ & $\mathbf{8}$ & $\mathbf{8}$ \\
\hline $\begin{array}{c}\text { soft } \\
\text { cross }\end{array}$ & $\begin{array}{c}\text { sharp } \\
\text { cross }\end{array}$ & wide \\
\hline
\end{tabular}

\begin{tabular}{|c|c|c|}
\hline y & $z$ & A \\
\hline $\mathbf{9}$ & $\mathbf{9}$ & $\mathbf{9}$ \\
\hline $\begin{array}{c}\text { open } \\
\text { counter }\end{array}$ & $\begin{array}{c}\text { close } \\
\text { counter }\end{array}$ & $\begin{array}{c}\text { straight } \\
\text { loop }\end{array}$ \\
\hline
\end{tabular}

Figure 5 : The different versions of the digits originate in the typeface DejaVu Sans. Each of the numerals 1, 2, 3, 4, 5, 6, 7, 8 and 9 have been created in three different variants, each having only one visual feature different from another version of the same number. The variables relate to one of the focus areas described above.

\subsection{Experimental Protocol}

Each subject ran a single experimental session (total duration of the session: about 1 hour) to test his/her ability to identify each of the 27 digits in a crowded environment (digits surrounded by other digits) while using his/her peripheral vision. The session was divided into 6 experimental blocks of 100 trials each, 3 blocks of trials presented in the lower visual field and 3 blocks of trials presented in the right visual field. Figure 6 schematically describes the temporal course for each trial: observers were asked to fixate a dot centred on the screen. Gaze location was measured to control for steady fixation on the fixation target dot. When the subject was ready for the trial, he/she pressed the button on a hand-held joypad. This triggered an offset correction and initiated the trial: at $10^{\circ}$ eccentricity in the lower visual field, a string of three digits (three digits chosen randomly among the 27 possible ones with a standard inter-digit spacing) was briefly displayed for 150 milliseconds. The subject's answer (three numerals) was stored by the experimenter. We did not ask the subjects to identify which versions of the numerals were displayed. No pre- or post-masks were displayed before and after each display. The print size for each subject was obtained in a pre-test session so that the recognition rate was approximately $50 \%$ for the middle digit (print-size average: $0.78^{\circ}$, range: $0.74^{\circ}-0.83^{\circ 1}$ ). On average, each numeral was presented 67 times for each subject. Approximately $5 \%$ of the trials were discarded because of incorrect fixation. Note that similar to the figure example, different versions of the same numeral could be part of the same string.

\footnotetext{
${ }^{1} 0.74^{\circ}$ represents 20 pixels with our viewing distance and screen resolution.
} 


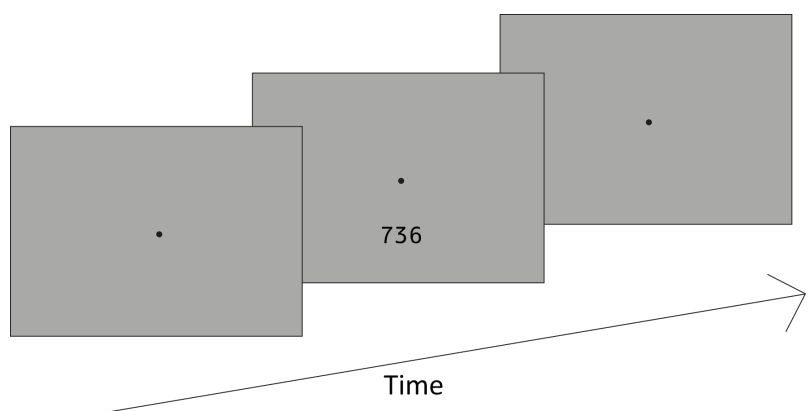

Figure 6 : Description of the experimental protocol: The subject fixated on a dot, pressed a button to display the string of 3 digits and then named the presented numeral.

\subsection{Statistical analysis of the individual digits}

Statistical analyses were performed using the R language and environment (Team, 2013). For each numeral from 1 through 9 , we investigated the effects of the different versions on recognition performance by using generalised linear mixed-effects models (function glmer of the Ime4 package). A model was run for each numeral (from 1 through 9). Random effect was the subject factor. Fixed effects were the version of the numeral (version 1, version 2, or version 3 ) and the position within the letter string (left, centre, or right letter). The dependent variable was the letter recognition error variable (0 or 1). P-values were based on conditional t-tests.

\subsection{Individual digits results}

Figure 7 shows the different recognition rates for each version of each numeral. First of all, numeral recognition rates can vary considerably across different numerals. For instance, the numeral ' 1 ' has an average recognition rate of $86 \%$ (average across the three different versions) whereas the numeral ' 8 ' has an average recognition rate of $56 \%$. This is due to letter confusion that exists only for some numerals. For example, on average, the digit ' 8 ' is confused with the numerals ' 5 ' or ' 6 ' $20 \%$ of the time, whereas the numeral ' 1 ' is confused with the numerals ' 5 ' or ' 6 ' less than $2 \%$ of the time, on average.

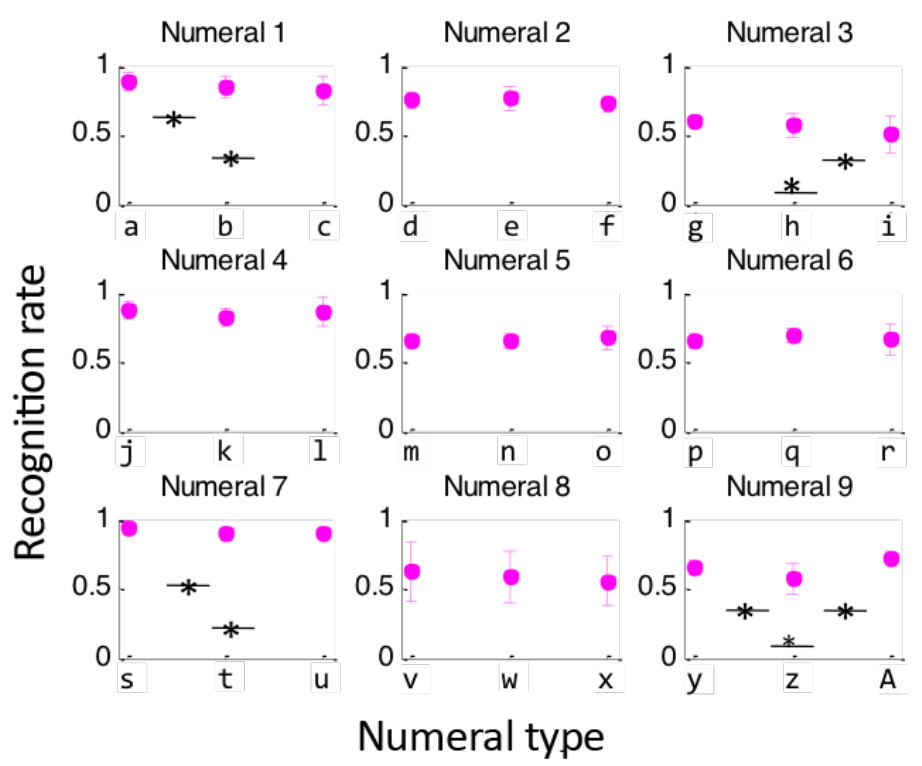

Figure 7 : Recognition rates for the different versions of each numeral 1-9. A star on the left represents a significant difference between version 1 and version 2 of the corresponding numeral. A star on the right represents a significant difference between version 2 and version 3 of the corresponding numeral. A centred star represents a significant difference between version 1 and version 3 of the corresponding numeral. 
For each numeral, our linear mixed-effect models show a significant effect of the relative position of the digit ( $p<0.00001$ for each model): The digit at the centre of the trigram is less often correctly identified, compared to the digits on the left or on the right of the trigram (53\% recognition on average for the central digit vs. $87 \%$ on average for the outside digits). This is because the magnitude of crowding depends on the number of flankers (Chanceaux, Mathôt, \& Grainger, 2014). More importantly this shows the significance of the differences between each pair of digits based on the different linear mixed-effect models. The pairs that are significant (across a same numeral) are also shown in Figure 7. Interestingly, our analysis shows that some versions of the numerals ' 1 ', ' 3 ', ' 7 ' and ' 9 ' are significantly easier to identify than other versions.

Table 1: P-values for numeral-pair comparisons. Significant differences are highlighted and marked with a star ( $p$ value<0.05). The yellow versions are the most legible.

\begin{tabular}{|l|l|l|l|}
\hline & 1 & 1 & 1 \\
\hline 1 & & $0.046^{*}$ & $0.0019^{*}$ \\
\hline 1 & $0.046^{*}$ & & 0.27 \\
\hline 1 & $0.0019^{*}$ & 0.27 & \\
\hline
\end{tabular}

\begin{tabular}{|l|l|l|l|}
\hline & 2 & 2 & 2 \\
\hline 2 & & 0.51 & 0.43 \\
\hline 2 & 0.51 & & 0.15 \\
\hline 2 & 0.43 & 0.15 & \\
\hline
\end{tabular}

\begin{tabular}{|l|l|l|l|}
\hline & 3 & 3 & 3 \\
\hline 3 & & 0.32 & $0.00085^{*}$ \\
\hline 3 & 0.32 & & $0.018^{*}$ \\
\hline 3 & $0.00085^{*}$ & $0.018^{*}$ & \\
\hline
\end{tabular}

\begin{tabular}{|l|l|l|l|}
\hline & 4 & 4 & 4 \\
\hline 4 & & 0.13 & 0.99 \\
\hline 4 & 0.13 & & 0.12 \\
\hline 4 & 0.99 & 0.12 & \\
\hline
\end{tabular}

\begin{tabular}{|l|l|l|l|}
\hline & 5 & 5 & 5 \\
\hline 5 & & 1.00 & 0.42 \\
\hline 5 & 1.00 & & 0.42 \\
\hline 5 & 0.42 & 0.42 & \\
\hline
\end{tabular}

\begin{tabular}{|l|l|l|l|}
\hline & 6 & 6 & 6 \\
\hline 6 & & 0.24 & 0.79 \\
\hline 6 & 0.24 & & 0.15 \\
\hline 6 & 0.79 & 0.15 & \\
\hline
\end{tabular}

\begin{tabular}{|l|l|l|l|}
\hline & 7 & 7 & 7 \\
\hline 7 & & $0.039 *$ & $0.045^{*}$ \\
\hline 7 & $0.039 *$ & & 0.96 \\
\hline 7 & $0.045^{*}$ & 0.96 & \\
\hline
\end{tabular}

\begin{tabular}{|l|l|l|l|}
\hline & 8 & 8 & 8 \\
\hline 8 & & 0.22 & 0.07 \\
\hline 8 & 0.22 & & 0.58 \\
\hline 8 & 0.07 & 0.58 & \\
\hline
\end{tabular}

\begin{tabular}{|l|l|l|l|}
\hline & 9 & 9 & 9 \\
\hline 9 & & $0.040^{*}$ & $0.018^{*}$ \\
\hline 9 & $0.040^{*}$ & & $1.000012^{*}$ \\
\hline 9 & $0.018^{*}$ & $1.000012^{*}$ & \\
\hline
\end{tabular}

\subsection{Complexity analysis}

Previous research - focusing on the recognition of crowded symbols in the periphery - has showed that symbol complexity (theoretically, the number of visual features of a single symbol) has a deleterious effect on the recognition of adjacent letters (Bernard \& Chung, 2011). Visual complexity can be measured following different methods that are strongly intercorrelated (Wang, He, \& Legge, 2014). Here, we decided to use the length of each digit skeleton for a given print size of 20 pixels (see Figure 8). This was done by using a custom-written Matlab program and templates for our different digits. Complexity values for each symbol are shown in Table 2 . 


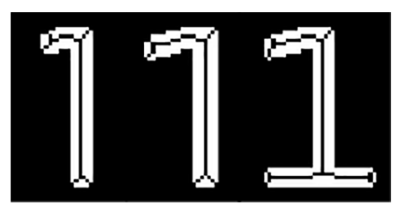

Figure 8: Symbol complexity. The complexity of each character is quantified by the length of each digit's morphological skeleton (example with a height of 41 pixels). The longer the string, the more complex the character.

For each presented digit (for instance the digit ' 2 ' within the trigram ' $123^{\prime}$ ), we studied (1) the effect of the complexity of the digit (i.e., the complexity of the digit ' 2 ') and (2) the effect of the complexity of the two adjacent digits (the sum of the complexity of the digit ' 1 ' and the complexity of the digit ' 3 '). To do so, we ran a new generalised linear mixed-effect model to study the effects of both kinds of complexity on recognition rate. Random effects were the subject factor and the numeral factor. Fixed effects were target complexity, flanker complexity, and the digit's position within the digit string (left, centre or right letter). The dependent variable was the digit recognition error variable (0 or 1$)$.

Table 2: Complexity values for the different versions of digits 1-9.

\begin{tabular}{|c|c|}
\hline Digit & Complexity \\
\hline 1 & 30 \\
\hline 1 & 33 \\
\hline 1 & 47 \\
\hline 2 & 44 \\
\hline 2 & 48 \\
\hline 2 & 46 \\
\hline 3 & 48 \\
\hline 3 & 51 \\
\hline 3 & 53 \\
\hline
\end{tabular}

\begin{tabular}{|c|c|}
\hline Digit & Complexity \\
\hline 4 & 54 \\
\hline 4 & 49 \\
\hline 4 & 63 \\
\hline 5 & 51 \\
\hline 5 & 52 \\
\hline 5 & 54 \\
\hline 6 & 53 \\
\hline 6 & 51 \\
\hline 6 & 54 \\
\hline
\end{tabular}

\begin{tabular}{|c|c|}
\hline Digit & Complexity \\
\hline 7 & 37 \\
\hline 7 & 37 \\
\hline 7 & 41 \\
\hline 8 & 58 \\
\hline 8 & 54 \\
\hline 8 & 63 \\
\hline 9 & 53 \\
\hline 9 & 56 \\
\hline 9 & 51 \\
\hline
\end{tabular}

\subsection{Complexity results}

The data show a significant effect of the complexity of the displayed target $\left(p<10^{-4}\right)$, and a significant effect of the complexity of the displayed flankers $\left(p<10^{-3}\right)$ on target recognition rate. The effect is stronger for target complexity $(-0.5 \%$ per skeleton pixel) than for flanker complexity $(-0.2 \%$ per skeleton pixel). To summarise, we found that for a given digit, the recognition rate significantly increases when the complexity of the digit decreases and the complexity of the adjacent digits decreases. Finally, the effect of trigram complexity (sum of the complexity of the three letters, i.e., the sum of both types of complexity) on trigram recognition rates is shown in Figure 9 . It clearly exhibits the negative effect of digit complexity on recognition rate based on the definition of six different ranges of complexity: [111:120], [121:130], [131:140], [141:150], [151:160] and [161:170]. There are at least 40 trials per subject for each complexity range. 


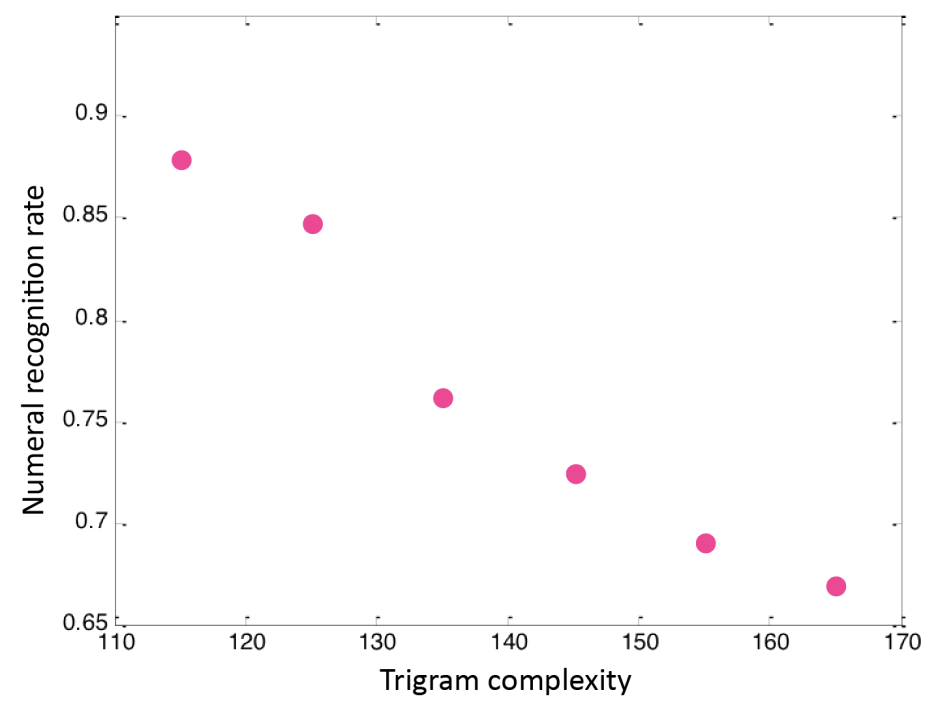

Figure 9: Effect of trigram complexity (sum of the length of the skeleton for the three digits) on numeral recognition rate. Recognition rate is averaged across subjects.

\section{Discussion}

In the following, we will compare the present findings (summarised in Figure 10) with data from other kinds of experimental designs and discuss the implications.
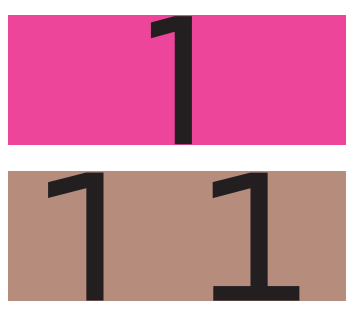
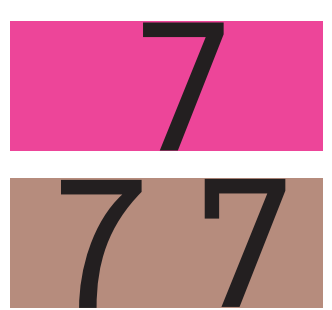
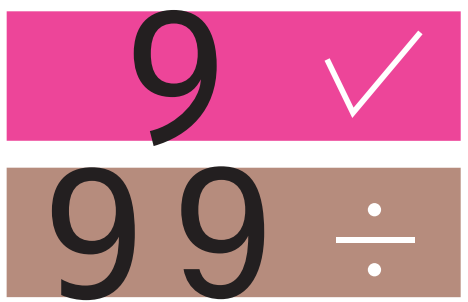

Figure 10: The characters of the top row were all found to be significantly more legible than the corresponding characters in the bottom row.

\subsection{The digit ' 1 '}

Our results showed that the narrow version of the digit ' 1 ' was more legible than the wider versions. The finding contradicts previous research by Fox et al. (2008), who investigated the legibility of single characters of 20 different typefaces and recommended a large surface area for the ' 1 ', so that the character is both tall and wide with a distinctive arm and a crossbar. While our focus is on three-digit strings, Fox et al. studied single characters. Furthermore, in our study the digits could only be misread for other digits, while the Fox et al. study also included letters and symbols as possible confusion material. We argue that except for the reading of codes, in most reading situations involving digits, possible confusion characters will be other digits. Hence the findings of Fox et al. cannot necessarily be translated into normal reading.

Our finding that narrowness benefits the digit ' 1 ', is supported by a previous study into reading distances, which found that serifs on the top and bottom of the stem resulted in a greater number of misreadings between the letters ' $i$ ' and 'I' (Beier \& Dyson, 2014). In another distance study, the results indicated that a serif on top without a large cross bar at bottom made the character more legible compared to one version with a cross bar and another sans serif version. However, the same study 
also concluded that narrow letters such as ' $\mathrm{l}$ ', ' $\mathrm{t}$ ', and ' $\mathrm{j}$ ' benefit from slightly wider designs, yet not too wide (Beier \& Larson, 2010).

It is possible that when characters we expect to be narrow lose their uniqueness as narrow, they become more difficult to identify, even though they might be easier to spot. Furthermore, while the alphabet includes several narrow letters that potentially can be misread for each other, there is only one narrow digit. Hence, the narrower the digit ' 1 ' the fewer misreadings for other digits.

\subsection{The digit ' 7 '}

The digit ' 7 ' was significantly more legible without a serif at the horizontal crossbar. The effect of serifs has been a central focus point throughout the history of legibility research. However, many of these studies lacked both internal and external validity as they often look for answers by comparing different typefaces (Lund, 1999).

By applying a method of Rapid Serial Visual Presentation of words, Morris et al. (2002) found that a sans serif version of the typeface Lucida was more legible in very small sizes at distance than a serif version of Lucida, the typefaces were designed for the study to control all other variables than the serif. The study by Beier and Dyson (2014) applied a similar approach of controlling other variables and found that single letters with serifs at the vertical extremes were more legible at great distances than sans serif letters (see Figure 11).

The different results confirm the notion that legibility-related findings identified under one reading condition cannot necessarily be translated into another reading condition. In other words, the higher error rate found in our study for the digit ' 7 ' with a serif might not be found if the same character is tested at greater reading distances or presented in isolation.

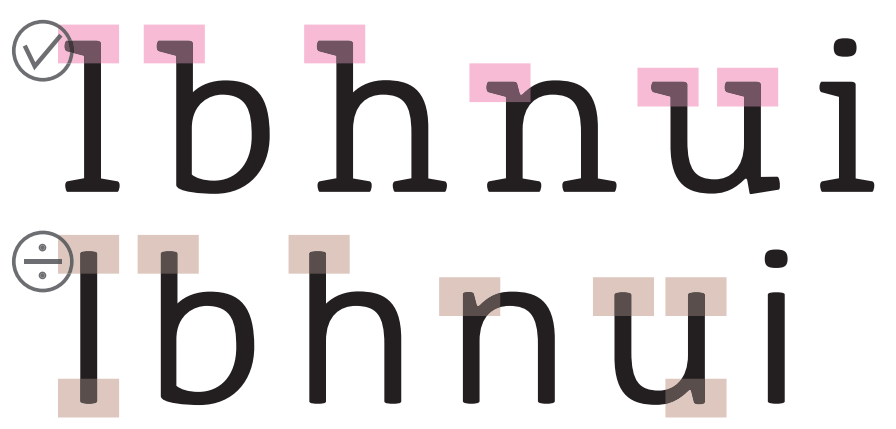

Figure 11: By measuring the maximum distance for the identification of single characters, Beier and Dyson (2014) found that serifs at the vertical extreme enhanced legibility.

\subsection{Digits ' 3 ' and '9'}

For the digits ' 3 ' and ' 9 ' our data indicates that versions with open apertures are more legible than versions with more closed apertures. This finding confirmed a widely voiced opinion by many type designers, who speak advocate the design of types with open inner counters, as they view this as a way to improve legibility (Kinneir, 1978, 1980; Unger, 2007). The central function of the open aperture is demonstrated by an experiment reported by Fiset et al. (2008). Here the researchers blurred different parts of the letters and found that subjects were better at identifying letters when the stroke endings were visible. For example, the stroke endings defining the open part of the ' $c$ ' are essential for distinguishing it from the letter ' $\mathrm{o}$ ' (Figure 12). Following this, we can conclude that the open apertures of digits ' 3 ' and ' 9 ' help to differentiate the characters from similar digits such as ' 8 ' and ' 6 '. 
In 2007, a team of researchers conducted an investigation in connection with the development of the road traffic typeface 'Tern' (Trans-European Road Network) (Smuc et al., 2007); in this study they compared the distance legibility of a range of different European traffic typefaces. Based on the data, the team recommended against closed counters and suggested that the digits ' 6 ' and ' 9 ' should have a curved tail.
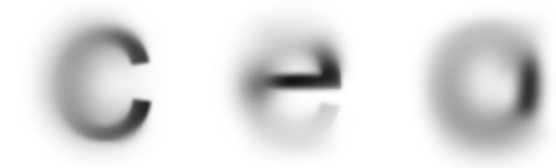

Figure 12: By blurring different parts of the letters, Fiset et al. (2008) found that letters where the stroke endings were visible were the easiest to identify (our illustration).

There are strong indications that open apertures benefit reading both within the peripheral visual field and for distance reading. However, that may not be the case when characters are seen in isolation. Recently, Larson and Carter (2016) published parts of the experimental research they had undertaken while developing the typeface Sitka. For a brief exposure within the central visual field, their findings suggest that letters with more open counters performed best when flanked by other letters; however, the study also found indications to suggest that letters with slightly closed counters performed best when viewed in isolation.

\subsection{Complex and simple skeletons}

The data revealed that strings of numerals with simple morphological skeletons were more legible than strings of numerals with more complex morphological skeleton (Figure 9) when numerals were presented within the peripheral visual field. This influence of symbol skeleton complexity on recognition rate was first demonstrated by Bernard \& Chung (2011), who tested the typefaces Times Roman and Courier and the script typefaces Edwardian and Aristocrat at an eccentricity of $10^{\circ}$ in the peripheral vision. They found that letter identification error rate increases with flanker complexity, up to a certain value.

This is an interesting finding in relation to the design of legible characters and numerals for subjects who cannot use their central vision. Yet, it somewhat contradicts the approach applied by several renowned type designers whose focus on ensuring differentiation between characters may result in added features, such as cross bars and tails (Herrmann, 2012; Johnston, 1913; Spiekermann, 2007). For the London Underground typeface, Johnston created a loop in the lowercase 'I' to differentiate the character from the capital ' $I$ ' (Walter, 1986). This resulted in a more complex letter skeleton, which in theory would lower legibility. However, the issue is not straightforward. As mentioned above, Beier and Larson (2010) found that at greater reading distances, a tail on the 'I' results in fewer errors.

Further, a two-storey ' $a$ ' and ' $g$ ' also have more complex letter skeletons than single-storey versions. However, Beier and Larson (2010) established that the two-storey ' $a$ ' is more legible than the singlestorey ' $a$ ', as the latter produced a high number of misreadings, being confused with ' $\mathrm{o}$ ' and ' $\mathrm{q}$ ' (Figure 13).
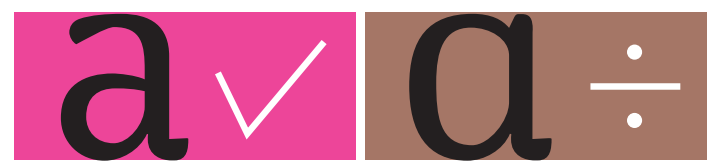

Figure 13: Beier and Larson (2010) found that with brief exposure and at distance reading, the two-storey ' $a$ " (left) was more legible than the single-storey ' $a$ ' (right). 
It appears that simple letter and digit skeletons generally improve legibility; however, this is only the case when the simplicity does not result in character shapes that are easily misread for others. Numerals are a great example of a set of symbols whose complexity can be significantly reduced: there are only 10 different symbols, and thus fewer confusion pairs compared to the Roman alphabets with their 26 or more characters. Based on the findings of this paper, we recommend the design of digit skeletons that follow the typeface of the top row in Figure 14, while the bottom row shows a typeface with less legible designs.
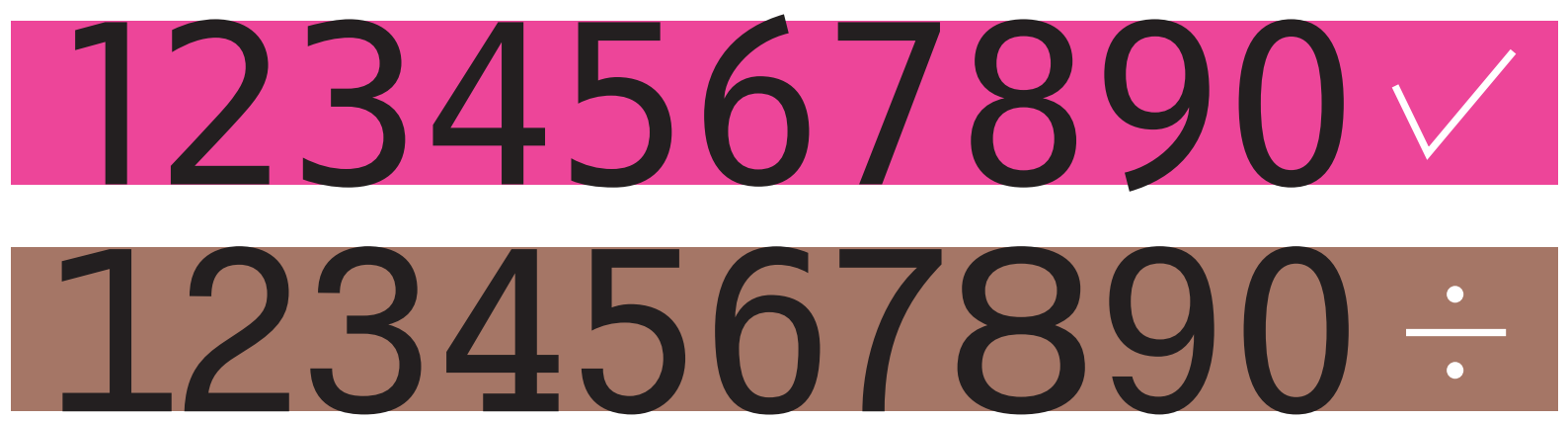

Figure 14: The digits in the top row were collectively more legible than the digits in the bottom row.

\section{Conclusion}

The purpose of this study was to identify the most legible digit skeletons for readers relying on their peripheral field of vision. The experiment produced significant findings for four out of the nine numbers tested. The results showed that the digit ' 1 ' should be narrow without a crossbar at the bottom, the digit ' 3 ' may either have open apertures or a triangular upper part, the digit ' 7 ' should be designed with a straight leg with no serif at the vertical bar, and the digit ' 9 ' should have open apertures with the bowl being somewhat straight and not too round.

The results further showed that a simple morphological digit skeleton facilitates greater peripheral legibility than more complexed skeletons by (1) increasing its own legibility and (2) increasing the legibility of adjacent digit skeletons. Based on previous research, it is argued that this is only relevant in situations where the simple digit skeleton will not result in a greater number of misreadings for other characters.

As the stimuli were designed for this specific experiment we were able to isolate the variable of digit skeleton for investigation, a methodological approach that improves the external validity of the findings and generates a set of usable 'rules of thumb' that can be easily implemented in the design of new typefaces.

Our results are highly relevant for the design of numerals that would be of specific benefit to individuals with central field loss, such as age-related macular degeneration. It has also been suggested that improved peripheral letter legibility could benefit reading performance for subjects who are able to use their central vision when they read running text (Rayner \& Pollatsek, 1989). Thus, our findings could also benefit normally sighted individuals.

\section{References}

Beier, S. (2016). Letterform Research: an academic orphan. Visible Language, 50(2), 64.

Beier, S., \& Dyson, M. C. (2014). The influence of serifs on 'h' and 'l': useful knowledge from design-led scientific research. Visible Language, 47(3), 74-95.

Beier, S., \& Larson, K. (2010). Design Improvements for Frequently Misrecognized Letters. Information Design Journal, 18(2), 118-137. 
Berger, C. (1944). I. Stroke-width, form and horizontal spacing of numerals as determinants of the threshold of recognition. Journal of Applied Psychology, 28(3), 208.

Bernard, J.-B., \& Chung, S. T. (2011). The dependence of crowding on flanker complexity and target-flanker similarity. J Vis, 11(8).

Carter, M. (2004). An Exercise in Versatility. In L. Cabarga (Ed.), Logo, Font \& Lettering Bible: A comprehensive guide to the design, construction and usage of alphabets, letters and symbols (pp. 200): Davis \& Charles.

Chanceaux, M., Mathôt, S., \& Grainger, J. (2014). Effects of number, complexity, and familiarity of flankers on crowded letter identification. J Vis, 14(6), 7-7.

Fiset, D., Blais, C., Ethier-Majcher, C., Arguin, M., Bub, D., \& Gosselin, F. (2008). Features for identification of uppercase and lowercase letters. Psychol Sci, 19(11), 1161-1168.

Fox, D., Chaparro, B. S., \& Merkle, E. (2008). Examining the Legibility of the Number" 1 " and the" $\div$ " Symbol. Usability news, 10(1).

Hamilton, J. P., Mirkin, M., \& Polk, T. A. (2006). Category-level contributions to the alphanumeric category effect in visual search. Psychon Bull Rev, 13(6), 1074-1077.

Herrmann, R. (2012). The Design of a signage typeface. Retrieved from http://ilovetypography.com/2012/04/19/the-design-of-a-signage-typeface/

Hess, R. F., Dakin, S. C., \& Kapoor, N. (2000). The foveal 'crowding'effect: physics or physiology? Vision Res, 40(4), 365-370.

Hind, P., Tritt, B., \& Hoffmann, E. (1976). Effects of level of illumination, strokewidth, visual angle and contrast on the legibility of numerals of various fonts. Paper presented at the Australian Road Research Board (ARRB) Conference, 8th, 1976, Perth.

Johnston, E. (1913). Writing \& illuminating, \& lettering: Macmillan.

Jonides, J., \& Gleitman, H. (1972). A conceptual category effect in visual search: O as letter or as digit. Attention, Perception, \& Psychophysics, 12(6), 457-460.

Kinneir, J. (1978). The practical and graphic problems of road sign design. In R. Easterby \& H. Zwaga (Ed.), Information design: the design and evaluation of signs and technical information (pp. 341-358): Chichester: John Wiley and Sons Ltd.

Kinneir, J. (1980). Words and buildings: the art and practice of public letterings. London: The Architectural Press.

Lansdell, H. (1954). Effects of form on the legibility of numbers. Canadian Journal of Psychology/Revue canadienne de psychologie, 8(2), 77.

Larson, K., \& Carter, M. (2016). Sitka: a collaboration between type design and science.

Legge, G. E., Klitz, T. S., \& Tjan, B. S. (1997). Mr. Chips: an ideal-observer model of reading. Psychol Rev, 104(3), 524.

Liu, L., \& Arditi, A. (2001). How crowding affects letter confusion. Optometry \& Vision Science, 78(1), 50-55.

Lund, O. (1999). Knowledge Construction in Typography: The case of legibility research and the legibility of sans serif typefaces. (PhD).

Marinus, E., Mostard, M., Segers, E., Schubert, T. M., Madelaine, A., \& Wheldall, K. (2016). A Special Font for People with Dyslexia: Does it Work and, if so, why? Dyslexia, 22(3), 233-244.

Morris, R. A., Aquilante, K., Yager, D., \& Bigelow, C. (2002). P-13: Serifs Slow RSVP Reading at Very Small Sizes, but Don't Matter at Larger Sizes. Paper presented at the SID Symposium Digest of Technical Papers.

Pelli, D. G., \& Tillman, K. A. (2007). Parts, wholes, and context in reading: A triple dissociation. PLoS One, 2(8), e680.

Polk, T. A., \& Farah, M. J. (1998). The neural development and organization of letter recognition: Evidence from functional neuroimaging, computational modeling, and behavioral studies. Proceedings of the National Academy of Sciences, 95(3), 847-852.

Rath, D., Domahs, F., Dressel, K., Claros-Salinas, D., Klein, E., Willmes, K., \& Krinzinger, H. (2015). Patterns of linguistic and numerical performance in aphasia. Behavioral and Brain Functions, 11(1), 2.

Rayner, K., \& Pollatsek, A. (1989). The Psychology of Reading: Lawrence Erlbaum Associates.

Schubert, T. (2016). Why are digits easier to identify than letters? Neuropsychologia.

Smuc, M., Windhager, F., Siebenhandl, K., \& Egger, S. (2007). Impaired Visibility Typeface Test-Report. Report In-Safety $A, 2$.

Spiekermann, E. (2007). How does the serif on a sans-serif $\mathrm{i}$ increase legibility/readability? ( Comment in the discussion ). Retrieved from http://typophile.com/node/38217 
Starrfelt, R., \& Behrmann, M. (2011). Number reading in pure alexia-A review. Neuropsychologia, 49(9), 22832298.

Team, R. D. C. (2013). R: A language and environment for statistical computing $R$ Foundation for Statistical Computing, Vienna, Austria http://www.R-project.org.

Unger, G. (2007). While You're Reading, . New York: Mark Batty Publisher.

Van Nes, F. L., \& Bouma, H. (1980). On the legibility of segmented numerals. Human Factors: The Journal of the Human Factors and Ergonomics Society, 22(4), 463-474.

Walter, T. (1986). Letters of credit (a view of type design): Gordon Fraser, London.

Wang, H., He, X., \& Legge, G. E. (2014). Effect of pattern complexity on the visual span for Chinese and alphabet characters. J Vis, 14(8), 6-6.

About the Authors:

Sofie Beier is a type designer and associate professor specialised in typeface legibility. She is the author of the books 'Reading Letters: Designing for legibility' and 'Type Tricks: Your personal guide to type design'.

Jean-Baptiste Bernard is a postdoctoral researcher specialized in the visual aspects of reading. His recent works concern the study of visual factors limiting letter recognition, word recognition and reading in normal and peripheral vision.

Eric Castet is a CNRS research director specialized in visual perception and eye movements at the Laboratoire de Psychologie Cognitive. His recent works concern the influence of visuo-attentional and psycho-linguistic factors that determine reading performance in normal and low vision. 